\title{
ERRATUM
}

S. Péché

\section{The largest eigenvalue of small rank perturbations of Hermitian random matrices}

Accepted: 20 September 2005 /

Published online: 10 November 2005 - (C) Springer-Verlag 2005

Unfortunately two author corrections were not carried out. The correct formulas are shown below.

1. page 30 : line -3 : the formula should be

$$
\text { Then } K_{N, 1}^{\prime}(x, y)=\frac{k_{N}}{(2 i \pi)^{2}} \int_{\Gamma^{\prime}} \int_{\gamma} h(s, t) g(s, t) e^{\left\{-k_{N} K_{y}(s)+k_{N} K_{y}(t)\right\}} d s d t \text {. }
$$

2. page 32 formula (82) should appear as

$$
\begin{aligned}
& \exp \left\{-N F_{u}(1)+N F_{v}\left(\pi_{1}+\alpha_{N} t_{N}\right)\right\} \\
& \quad=\frac{\exp \left\{N\left(\left(\pi_{1}+\alpha_{N} t_{N}\right)^{2} / 2-C\left(\pi_{1}\right)\left(\pi_{1}+\alpha_{N} t_{N}\right)\right)\right\}}{\exp \left\{N\left(1 / 2-C\left(\pi_{1}\right)\right)\right\}}\left(\pi_{1}+\alpha_{N} t_{N}\right)^{N} \\
& \times\left[\exp \left\{N\left(C\left(\pi_{1}\right)-v\right)\left(\pi_{1}+\alpha_{N} t_{N}\right)-N\left(C\left(\pi_{1}\right)-u\right)\right\}\left(\pi_{1}+\alpha_{N} t_{N}\right)^{-k_{N}}\right. \\
& \left.\times\left(\frac{\alpha_{N} t_{N}}{1-\pi_{1}}\right)^{k_{N}}\right]
\end{aligned}
$$

The online version of the original article can be found at http://dx.doi.org/10.1007/s00440-005-0466-z 